\title{
MERCADO DE TRABAJO E INMIGRACIÓN \\ EN LA I GLOBALIZACIÓN: URUGUAY Y COMPARACIONES REGIONALES
}

María M. CaMou*

doi.org/10.47003/RUHE/10.17.01

\section{Resumen}

El objetivo de esta investigación es estudiar las relaciones de trabajo en la región del Cono Sur y Bolivia durante la Primera Globalización. Se indaga especialmente en el caso de Uruguay y el rol de la inmigración en este proceso. Nuestra hipótesis es que la misma juega un rol determinante en las características del mercado de trabajo y su originalidad respecto a otros países de la región. Nuestro período de estudio comprende desde finales del siglo XIX y principios del XX. En este marco de tiempo, se consolida gradualmente en la región un mercado de trabajo con una oferta de trabajo más estable y en expansión. Mientras que en Uruguay y en Argentina se observa un incipiente mercado de trabajo "moderno" con una importante proporción de trabajo mercantilizado, Bolivia y Brasil muestran una estructura distinta con la predominancia de formas de trabajo no asalariado.

Palabras clave: mercado de trabajo, inmigración, Uruguay, globalización

\begin{abstract}
The objective of this research is to study the evolution of labor relations for the Southern Cone region and Bolivia during the First Globalization. Then the paper focus on the case of Uruguay and the role of immigration in this process. Our hypothesis is that immigration plays a decisive role in the characteristics of the labor market and its originality with respect to other countries in the region. Our study period includes from the late nineteenth and the early twentieth centuries. In this period, a labor market gradually consolidated in the region with a labour supply that increases and becomes more stable. Salaried work became a significant proportion of workers but this process was very heterogeneous among the different observed countries.
\end{abstract}

Keywords: labour market, inmigration, Uruguay, Globalization

*maria.camou@cienciassociales.edu.uy

Programa de Historia Económica y Social. Universidad de la República 


\section{Introducción}

El objetivo de esta investigación es estudiar la evolución de las relaciones de trabajo para la región del Cono Sur y Bolivia durante la Primera Globalización. Nuestro período de estudio comprende desde finales del siglo XIX hasta comienzos del XX. En este marco de tiempo, se consolida gradualmente en la región un mercado de trabajo con una oferta de trabajo que aumenta y se vuelve más estable. El trabajo asalariado se constituye en una proporción significativa de los trabajadores.

Si tomamos en cuenta la comparación entre los países observados encontramos que el proceso es muy heterogéneo Se indaga especialmente en el caso de Uruguay y el rol de la inmigración en este proceso. Nuestra hipótesis es que en Uruguay la inmigración juega un rol determinante en las características del mercado de trabajo y lo diferencia de otros países de la región. La metodología y el procesamiento de los datos para estudiar la evolución en la región y su comparación con el resto de los países latinoamericanos está basada en la elaborada por Global Collaboratory sobre la historia de las relaciones laborales, 1500-2000. La clasificación utilizada en esta base de datos permite analizar la interacción entre las relaciones laborales y demográficas, teniendo en cuenta la relación con el trabajo de todos los sectores de la población: activos e inactivos.

Una de las ventajas de esta metodología y del acervo de datos que ha generado el proyecto Global Collaboratory es la incorporación de todas las formas que adopta la relación laboral, a saber, los productores no asalariados y familiares, las personas sin actividad laboral declarada, los desocupados, los patrones, etc. A nuestro entender, esta taxonomía es especialmente adecuada especialmente para analizar la etapa de consolidación del mercado de trabajo y los cambios que se producen a lo largo del período en Uruguay y en América Latina.

La conformación del mercado de trabajo aparece en la visión dominante de la economía y de la historia hasta la década de los sesenta como el pasaje de trabajo auto sustentando al trabajo asalariado, en una evolución en etapas, fuertemente determinada por la demanda y los cambios en la estructura productiva. Investigaciones más recientes han cuestionado está mirada unilateral y consideran que deben tomarse en cuenta otros factores tales como el espacio, las características de la población, la estructura productiva y las instituciones en el desarrollo del mercado de trabajo (Grantham 1994).

Desde la perspectiva del proyecto de Ámsterdam el trabajo adquiere, a lo largo de la historia, diferentes formas, la mayoría de ellas no remuneradas y experimenta cambios, pero no en un sentido estrictamente progresivo, sino que tanto el trabajo asalariado como la esclavitud y la servidumbre han aparecido y desaparecido en diferentes etapas. Otro aspecto que se resalta es que las condiciones de trabajo y las remuneraciones no son únicamente producto del resultado de las fuerzas de mercado sino también de las acciones individuales y colectivas de los trabajadores (Lucassen 2013).

Con esta idea de estudiar la relación con el trabajo de toda la población es que hemos optado por esta metodología que muestra los cambios en la inserción de la población y de sus distintos grupos (mujeres, inmigrantes) en las relaciones de trabajo. La comparación entre el caso de Uruguay y Argentina, con una estructura poblacional marcada por la inmigración, y otros países de la región con diferentes procesos de población, permite analizar la interacción entre población, espacio o territorio y relaciones de trabajo.

\section{Metodología y fuentes}

En esta investigación nos proponemos explorar el mercado de trabajo en el cambio de siglo XIX al XX. Para América Latina, este fue un período de consolidación progresiva del mercado laboral, el fin de la esclavitud y un aumento de la proporción de asalariados en la fuerza laboral. Al mismo tiempo, algunos de los países observados estaban comenzando su transición demográfica.

En primer lugar, comparamos las relaciones de trabajo para algunos países de la región en el pasaje del siglo XIX al siglo XX. Tratando de explicar la especificidad del caso uruguayo, en la segunda parte del artículo se profundiza en los cambios que experimenta. Para Uruguay la información disponible permite analizar las diferencias entre distintos grupos que conforman el mercado de trabajo, principalmente inmigrantes y nacionales, pero también los cambios a lo largo del tiempo, tomando dos observaciones puntuales $1858 / 59$ y 1908 .

Consideramos que la metodología empleada por el proyecto Global Collaboratory on the History of Labour Relations, 1500-2000, del Instituto Internacional de Historia Social (IISH) es especialmente 
adecuada para estudiar esta etapa de formación del mercado de trabajo y de importantes cambios demográficos.

Por esta razón nuestro análisis de las relaciones laborales en América Latina se basa en los criterios de clasificación de la base de datos de este proyecto. La misma recopila información de los diferentes países en torno a cada cambio de siglo en el periodo 1500-2000.

La taxonomía empleada implica tomar en consideración no solamente a los individuos sino también hogares, instituciones políticas y mercado. Cada individuo de una población es ordenando en distintos grupos según su relación con el trabajo.

Como punto de partida para cada unidad geográfica y sección transversal, tomamos a toda la población y posteriormente determinamos qué parte no trabaja como regla y, en consecuencia, qué parte trabaja (estos "cálculos" a menudo se basarán en estimaciones en lugar de datos precisos).

Presentamos a continuación la clasificación empleada, aunque en nuestro caso y especialmente en nuestro período, algunas categorías no se encuentran representadas, especialmente el subgrupo III correspondiente al trabajo tributario.

\section{Población no trabajadora, inactivos:}

1. No pueden trabajar o no puede esperarse que trabajen: porque son demasiado jóvenes $(\leq 10 / 14$ años), demasiado mayores ( $\geq 70$ años), discapacitados o estudiantes.

2. Rentistas: no necesitan trabajar porque tienen otros ingresos (rentistas, pensionistas, etc.)

3. Desempleados: aunque el desempleo es en gran medida un concepto del siglo XIX y, especialmente, del siglo XX, distinguimos entre los que tienen empleo y los que desean trabajar pero que no pueden encontrar empleo.

\section{Trabajo familiar/recíproco:}

Las personas que proporcionan mano de obra para otros miembros del mismo hogar y/o comunidad.

4. Productores autoconsumo: jefes de hogares autosuficientes

5. Familiares subordinados a.o. cónyuges (hombres y mujeres) e hijos de los jefes de hogares autosuficientes que contribuyen al mantenimiento del hogar realizando un trabajo productivo. Hemos seguido el criterio de incluir a la población adulta sin ocupación dentro de una familia viviendo en un hogar (excluyendo al jefe de familia) en esta categoría.

6. Servidores domésticos y esclavos que contribuyen al mantenimiento de hogares autosuficientes.

7. Trabajadores para la comunidad: personas que realizan tareas para la comunidad local a cambio de una remuneración en especie, tales como alimentos, alojamiento y servicios, o una parcela de tierra

\section{Trabajo tributario:}

Personas que están obligadas a trabajar para instituciones (a menudo el Estado, aunque también podría ser para una autoridad feudal o religiosa). 8. Trabajadores forzados 9. Trabajadores tributarios contratados 10. Siervos tributarios.11. Esclavos tributarios.

\section{Trabajo mercantilizado}

12. Autónomos: aquellos que producen bienes o servicios para instituciones de mercado, posiblemente en cooperación con otros miembros del hogar o con un máximo tres trabajadores asalariados, aprendices, siervos o esclavos. La identificación de este grupo resulta a veces difícil ya que los datos censales definen el oficio o profesión de la persona, pero no se especifica si se trata de un trabajador independiente o dependiente. En algunos casos se cruzó la información de los censos de población con los censos industriales y comerciales para identificar mejor a este grupo.

13. Empleadores: aquellos que producen bienes o servicios para instituciones de mercado mediante el empleo de más de tres trabajadores asalariados, trabajadores por contrato, siervos o esclavos.

14. Asalariados: que producen bienes o servicios para el mercado a cambio principalmente de una remuneración monetaria.

15. Trabajadores: aquellos contratados para trabajar como trabajadores no libres para un empleador por un período específico de tiempo para pagar una deuda privada.

16. Siervos que trabajan para el mercado.

17. Esclavos que producen para el mercado: los que son propiedad de sus empleadores (amos). Ins- 
tituciones no comerciales.

18. Asalariados empleados por instituciones no comerciales (que pueden o no producir para el mercado) tales como el Estado, las empresas estatales, la Iglesia o las cooperativas de producción, que producen o prestan servicios para un mercado libre o regulado

Los países en nuestra muestra están limitados a aquellos para los cuales la información está disponible en la base de datos del proyecto Global Collaboratory, a saber, Argentina, Brasil y Bolivia. Los datos clasificados según esta tipología para estos tres países latinoamericanos fueron procesados por investigadores del proyecto Global Collaboratory, oportunamente citados. Sería muy desaeable que futuras investigaciones ampliaran el espectro territorial y cronológico de estas bases de datos.

La distancia temporal entre los censos observados para los diferentes países no es menor, pero cumple el cometido de abarcar el período de la I Globalización que nos proponemos abordar.

Hemos construido para el caso de Uruguay dos observaciones $1858 / 59$ y 1908.

La muestra que tenemos es interesante y nos permite capturar parte de la diversidad de América Latina. Al incluir Argentina y Uruguay, cubrimos países con una alta proporción de inmigrantes europeos, y con Brasil y Bolivia incorporamos países con una alta proporción de personas de ascendencia africana (ex esclavos) y grupos indígenas en sus poblaciones. Otra diferencia entre los países de la muestra es la distribución variable de las poblaciones urbanas y rurales, una variable vinculada a los orígenes étnicos de las personas. Las poblaciones predominantemente rurales en América Latina están asociadas con una alta proporción de personas indígenas, lo que significa que existen patrones culturales y familiares específicos, formas de control social en las comunidades y tipos de relaciones laborales.

Para Uruguay se utilizaron para la comparación regional datos para todo el país. En la segunda parte la unidad de análisis es Montevideo, centro principal de recepción de inmigrantes. En el primer año observado (1858) las fuentes son las hojas de encuesta levantadas para preparar el censo de 1860. Los datos recopilados incluyen edad, sexo, estado civil y lugar de nacimiento. La información sobre el segundo período (1908) se basa en el Censo General de la República (Censo General de la República), que fue el primer censo uruguayo con datos confiables a nivel nacional. El censo de población abarcó todo el país.

Para la clasificación de los distintos grupos en las relaciones se utilazaron criterios similares en 1858/59 y 1908. En el padrón de Montevideo de 1858/59 se incluyeron en la categoría "autónomo" profesiones tales como: barbero, artesano, carnicero, herrero, hojalatero, impresor, panadero, sastre, etc. Entre los empleadores se encuentran: propietarios, estancieros, dueño de fonda y diversas profesiones liberales. El grupo de trabajadores asalariados comprende entre otros: changador, dependiente, cocinero, conchabado, empleado, cigarrero, albañil, etc.

Los censos latinoamericanos de este período tienen muchas limitaciones. Para la mayoría de los países estas limitaciones dependían de intereses políticos inmediatos. Por otra parte, la limitante de los recursos financieros impedía mantener la periodicidad.

Para algunos países la delimitación del territorio fue una carencia importante, que se explica, entre otras razones, debido a la falta de mapas confiables de todas las regiones. Los procedimientos utilizados para realizar los censos presentaban carencias relevantes. La mayoría de los países ya utilizaban métodos de enumeración basados en el individuo en cuestión, pero otros países recopilaban la información de las respuestas proporcionadas por el jefe de familia, lo que probablemente daba lugar a errores y distorsiones en la información.

Teniendo en cuenta las características de estos censos en América Latina, señalaremos las deficiencias más importantes que deberán tomarse en cuenta al analizar los resultados.

En primer lugar, el número de personas ocupadas probablemente se sobreestima. Los censos registran la profesión o el puesto de trabajo de una persona sin definir si están o no empleados en ese momento. El concepto actual de "desempleado" no parece ser linealmente aplicable para esta etapa en la que el trabajo asalariado no era predominante. Sin embargo, su impacto no habría sido tan relevante en la medida en que se trata de un período de rápido crecimiento económico con una demanda creciente de mano de obra y un desempleo muy bajo.

La información recogida contiene muchas inexactitudes con respecto a las ocupaciones. En la introducción del censo uruguayo de 1908 por ejemplo se aclara:

"La pequeñez de las cifras correspondientes a los servicios estatales es atribuible a lo siguiente: muchos empleados no especificaron si eran públicos o privados; no pocos, además del empleo público, 
ejercían una profesión secundaria y, a menudo, se declararon solo estos últimos en el censo; y otros empleados son listados como profesionales (educación, justicia, etc.)".

En segundo lugar, la participación femenina en el mercado laboral es difícil de reconstruir dada la escasez de fuentes. Puede suponerse una subestimación de esta, debido a las técnicas de registro utilizadas y a los preconceptos de los funcionarios del censo.

En general, el trabajo femenino no ha sido bien documentado. Los censos realizados en las primeras décadas del siglo XX contienen incoherencias, como las diferencias en los criterios para registrar la participación de las mujeres en el sector primario. Como resultado la población total de trabajadores en este sector cambia, con frecuencia, atípicamente de un censo al otro.

El tercer aspecto a resaltar es el de la nacionalidad, especialmente importante para países que recbieron grandes contingentes migratorios. Países como Argentina y Uruguay definieron la nacionalidad según el jus solis. Es decir, todos los nacidos en el territorio nacional adquieren la nacionalidad. Sin embargo, en este período, países como Canadá y Estados Unidos que comparten este principio jurídico, optaron por registrar la nacionalidad de los ancestros, herramienta que les permitió conocer con mayor información el impacto de la migración en la sociedad. Según Otero (2011), para el caso argentino el no registro de la nacionalidad de los ancestros está ligado al minimalismo metodológico que impregnó la estadística de gran parte de los países latinoamericanos y tuvo como consecuencia una homogenización de la población nativa. Esta homegenización estadítica permea y a la vez abona a la tesis de un rápido "crisol de razas".

\section{Poblamiento y mercado de trabajo en la 1era. Globalización.}

A fines del siglo XIX y principios del XX, los países latinoamericanos experimentaron grandes cambios derivados de su inserción en la economía internacional. El sector exportador, apoyado por la modernización del transporte, impulsó el auge económico. A partir de esta expansión de la economía, se fueron conformando nuevas fronteras y regiones.

En el marco del crecimiento económico y la mayor estabilidad política América Latina se convierte en una región receptora de importantes contingentes migratorios. Como consecuencia de estos cambios se produjo un gran aumento de la población en el continente en su conjunto. Entre 1870 y 1930, la población latinoamericana pasó de representar un 2,9 a 4,2 por ciento del total de la población mundial. Pero este aumento de la población no estuvo distribuido homogéneamente entre los distintos países de América Latina. Bértola y Ocampo (2012) distinguen tres grupos de países acorde al ritmo de crecimiento de la población en el periodo 1870-1930. Bolivia se encuentra en el grupo de menor crecimiento de la población (aprox. 1\%), Brasil en el grupo intermedio (2\%) y Argentina y Uruguay en el tercer grupo de los países en que la población crece más rápidamente (3\%). En este proceso intervienen básicamente tres variables demográficas: fecundidad, mortalidad y migraciones.

En los países de nuestra muestra, Bolivia, con una fuerte herencia colonial y una estructura económica más o menos inalterada durante el período basada en grandes haciendas y minas, la población creció lentamente. Si bien no existen datos en esa época, la muy baja esperanza de vida (39 años) en 1950 hace suponer una aún más baja a comienzos del siglo XX. Por otra parte las migraciones internacionales fueron prácticamente inexistentes (Pérez 2017).

En Brasil, pese a la alta mortalidad y las precarias condiciones de vida, la esclavitud en una primera etapa y luego las oleadas migratorias de fines del siglo XIX contribuyeron a un moderado crecimiento poblacional.

Pero fundamentalmente, Argentina y Uruguay, países de clima templado y mayor crecimiento económico durante el periodo, fueron los que recibieron mayores flujos migratorios, respecto al tamaño de su población local.

Las migraciones hacia América Latina se concentraron en el último cuarto del siglo XIX, varias décadas más tarde que los flujos migratorios hacia el Norte de América, y estaban constituidas por inmigrantes que llegaron a estas costas procedentes de las regiones menos desarrolladas de Europa occidental (Hatton y Williamson, 1994). 
Cuadro 1. Tasa de inmigración en los países de la muestra, 1871-1910

\begin{tabular}{|l|c|c|c|c|}
\hline & ARGENTINA & BRASIL & BOLIVIA & URUGUAY \\
\hline $1871-1880$ & 124 & 20 & & 281 \\
\hline $1881-1890$ & 292 & 41 & & 248 \\
\hline $1891-1900$ & 163 & 71 & 4 & 114 \\
\hline $1901-1910$ & 311 & 34 & & 21 \\
\hline
\end{tabular}

Fuentes: Argentina, Brasil y Uruguay (Sanchez Alonso 2006), Bolivia (Censo de la República de Bolivia 1900).

A diferencia de la migración rural-urbana europea o de la migración del Viejo Mundo a los Estados Unidos, compuestas por agricultores y artesanos que viajaban con sus grupos familiares, las migraciones de finales del siglo XIX hacia América Latina, estaban conformadas predominantemente por varones jóvenes, solteros y no calificados. Los mayores flujos de migración al Río de la Plata y Uruguay pertenecen a esta etapa y comparten estas características demográficas.

Los migrantes que se establecieron en Argentina y Uruguay tenían diversos orígenes regionales y sociales, y las tradiciones culturales que trajeron con ellos respondieron a diferentes modelos de sociedad. La ruptura drástica causada por la migración al Nuevo Mundo significó un quiebre con el lugar de origen y un esfuerzo por adaptarse a una nueva sociedad. Una particularidad de los migrantes es que tienden a ser personas más dinámicas que la media de la población en sus lugares de origen.

Muchos investigadores han señalado que en ese momento América Latina era una región con escasez de mano de obra (Bulmer Thomas 2003), pero sería más preciso decir que la movilidad de los asalariados era escasa. El tipo de relaciones de trabajo heredadas de la época colonial como el trabajo coercitivo en regiones con predominancia de la población indígena y campesina como Bolivia, el legado de las relaciones sociales preexistentes como la servidumbre por deudas o el "trabajo de enganche" y la herencia de la esclavitud también constituyeron trabas para la formación de un mercado de trabajo moderno con predominio del trabajo asalariado.

En los países de economía templada, más integrados al comercio internacional, como Argentina y Uruguay, el mercado laboral y el trabajo asalariado se incrementaron hacia finales del siglo XIX. Pero también en estos países la consolidación del mercado de trabajo fue un proceso que duró varias décadas. Sábato y Romero distinguen en el periodo 1859-1880 diferentes formas de trabajo prexistente que no eran "libres" en Buenos Aires. Entre estas los trabajos forzados de los convictos, de aprendices y sirvientes. Estos tipos de relaciones laborales también estuvieron presentes en Montevideo (Camou y Pellegrino 1992). Sin embargo, en la medida en que estos países desarrollan un modelo agroexportador integrado a la economía mundial se impone gradualmente el trabajo asalariado en la región.

Investigaciones recientes confirman que la sociedad que se conforma en este período presenta altos niveles de desigualdad. Bértola (2000) estimó la evolución del índice de Gini para Uruguay en el período 1870-2000 y encontró que la desigualdad en el país empeoró entre 1870 y 1910. Este fue un período en el que los precios de la tierra aumentaron considerablemente más fuertemente que los salarios y la propiedad de la tierra tendió a una mayor concentración. También entre los trabajadores aumentó la desigualdad en la medida en que se incrementaba el peso relativo de la mano de obra no calificada, que incluía a muchos inmigrantes, mientras que la mano de obra calificada continuó siendo escasa. La inserción de inmigrantes en este período estuvo también condicionada por la restricción de tierra disponible para trabajar y el alto costo de las viviendas en las zonas urbanas.

El hecho de que hubiera grupos de inmigrantes en estos países agrega otra dimensión al estudio de la estructura social y las relaciones laborales. El tema de los grupos que mantuvieron su propia identidad nacional o étnica está relacionado con su posición en los diferentes estratos socioeconómicos, pero hay factores importantes que actúan como barreras limitantes en esta interrelación, como la resistencia de los grupos nativos a incorporar "extranjeros" y las luchas defensivas de grupos étnicos o nacionales para mantener su identidad. 


\section{Las relaciones de trabajo en América Latina en 1900}

Aplicando la taxonomía antes explicada, en 1900 encontramos relaciones de trabajo fuertemente diferenciada entre los países de la muestra.

Cuadro 2. Relaciones de trabajo por subgrupos (en \%)

\begin{tabular}{|l|c|c|c|c|}
\hline & BOLIVIA & ARGENTINA & URUGUAY & BRASIL \\
\hline TrABAJO MERCANTILIZADO & 27 & 48 & 33 & 18 \\
\hline TrABAJO FAMILIAR/ RECÍpROCO & 58 & 23 & 36 & 46 \\
\hline No TRABAJAN & 16 & 28 & 31 & 36 \\
\hline & 100 & 100 & 100 & 100 \\
\hline
\end{tabular}

Fuentes: Elaboración propia con base en https://iisg.amsterdam/en/research/projects/global-collaboratory-onthe-history-of-labour-relations-1500-2000 y Censo Nacional de 1908 (Uruguay)

Si se observa la distribución de la población ocupada por grandes grupos encontramos estructuras con diferencias importantes. El grupo de los que no trabajan, compuesto por niños, adultos mayores, rentistas y desocupados es muy reducido en Bolivia. La categoría de menores en el caso de Bolivia comprende hasta los 7 años, a diferencia del resto de los países donde se considera a los menores de 10. Esto está determinado por la forma en que fueron recolectados los datos y no admite otro tipo de desagregación. Por otra parte, se considera que la inclusión de este grupo de menores de entre 6 y 10 años estaría basada en su mayoritaria participación laboral. A su vez, el bajo peso de los inactivos en Bolivia puede estar también reforzado por características demográficas como la menor esperanza de vida y mayor mortalidad infantil y al subregistro de los menores bastante frecuente en los primeros censos.

Brasil en el otro extremo presenta una estructura de población en crecimiento con gran peso de los menores de edad.

Los otros dos grandes subgrupos de la taxonomía toman en cuenta por un lado el trabajo mercantilizado y por otro las diferentes formas de economía familiar, incluyendo a los productores rurales.

Bolivia, como sería esperable, presenta una estructura con mayor predominancia de pequeños agricultores y campesinos dedicados a la producción de subsistencia. Es también el país con más altos índices de población rural, 85.4 \% de la población en 1900. Como destacan Bértola y Gerschunoff (2011) la predominancia del campesinado implicó que una gran parte de la población quedará excluida de la economía de mercado. La contribución indígena, tributo exigido a los indígenas, representaba entre un 31 y un $50 \%$ de los ingresos del Estado entre 1880 y 1910. En este contexto, el trabajo mercantilizado tuvo poco peso en la estructura.

Argentina y Uruguay fueron los países más "avanzados" de la región. Argentina en primer lugar y luego Uruguay reflejan un mayor peso del sector mercantilizado en la estructura. Las demandas de la producción, la integración a la economía internacional y la mayor presencia de inmigrantes en estos países determinaron una pirámide de población atípica con mayor concentración de la población en la edad activa. También las altas tasas de masculinidad propias de los migrantes impactaron en las altas tasas de participación en el mercado laboral (Camou y Pellegrino 1992, Sábato y Romero 1992). Asimismo, el crecimiento poblacional que tiene lugar en los países del Plata implica un aumento de la demanda en servicios y en la construcción de su infraestructura.

El caso de Brasil presenta particularidades con un fuerte componente de la economía familiar. Este grupo además de la población específicamente integrada a una economía familiar, abarca también un amplio margen de la población sin ocupación declarada, por lo que se supone puede encubrir trabajadores zafrales, artesanos o informales. Es importante destacar que el $72 \%$ de este grupo está constituido por mujeres.

La presentación más desagregada de los datos (Gráfico 1) muestra mayores diferencias entre los países. En el grupo de los trabajadores mercantilizados, Uruguay tiene el componente mayor de asalariados y luego Argentina. También fueron los países más avanzados de la región en términos de PIB (Camou 2016). 
Gráfico 1. Relaciones de trabajo en América latina

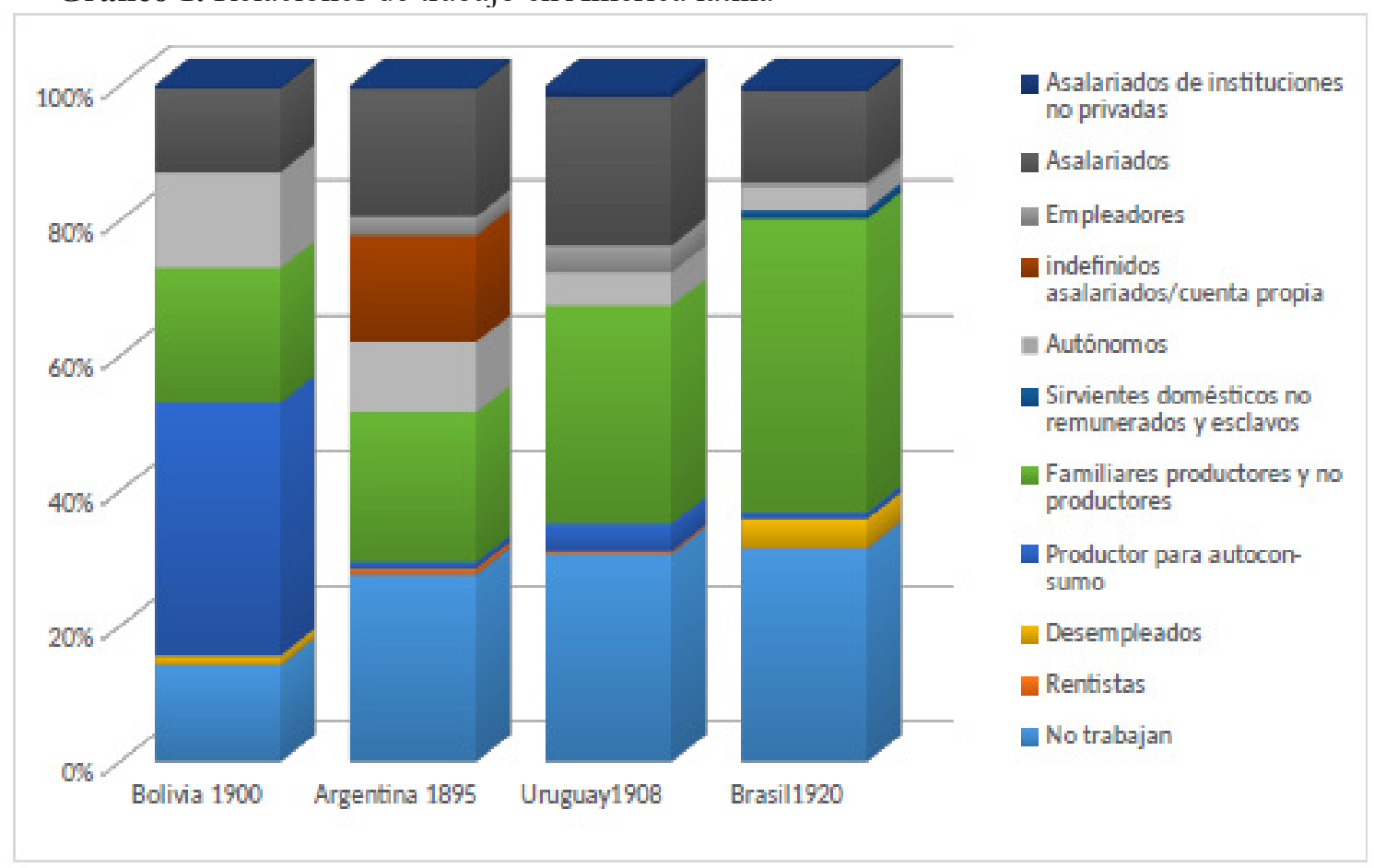

Fuente: Elaboración propia con base en https://iisg.amsterdam/en/research/projects/global-collaboratory-onthe-history-of-labour-relations-1500-2000 y Censo Nacional de 1908 (Uruguay)

Bolivia por el contrario tiene un grupo menor de asalariados a pesar de incluir a los menores entre 7 y 10 años y a una mayor cantidad de ocupaciones femeninas vinculadas a la producción textil artesanal.

El grupo de los autónomos o trabajadores independientes tiene el mayor peso en Argentina y en segundo lugar en Bolivia.

En este grupo para Argentina se realizaron algunas modificaciones a las estimaciones realizadas por Raquel Gil Montero (Gil Montero, n.d.). Los ocupados del comercio, profesiones liberales y artísticas fueron clasificados como autónomos. Los ocupados en la industria dada la descripción que incluye muchas profesiones que seguramente se ejercieron de forma independiente tales como zapatero, carnicero, panadero. Asimismo, tejedores, hilanderas, que suponemos en esa época se referían en parte a trabajo artesanal realizado en forma independiente o semi independiente fueron divididos en partes iguales entre asalariados y autónomos. Este subgrupo fue incluido en la categoría indefinidos asalariados/autónomos.

Otra diferencia remarcable es que, si bien en todos los países el Estado estaba en un proceso de consolidación incipiente, Uruguay presenta una mayor proporción de trabajadores en ese grupo.

\section{Uruguay: conformación demográfica y participación en el mercado laboral}

Analizar el caso de Uruguay nos permite profundizar en las características de la población y de las relaciones de trabajo en dos mojones en el tiempo que abarcan precisamente la etapa de consolidación del mercado de trabajo: 1858/59 y 1908. Los datos permiten también estudiar por separado el comportamiento de uruguayos y extranjeros en el mercado laboral.

En los dos períodos es notaria la diferencia de participación entre los hombres uruguayos y los extranjeros. Los inmigrantes presentan muy altas tasas de participación en el mercado de trabajo. Para 1908, esta diferencia perdura, pero tiende a disminuir (Camou, 1998). El perfil demográfico propio de los inmigrantes y de los nativos proporciona una explicación para gran parte de estas diferencias. 
Cuadro 3. Tasa de participación. Montevideo

\begin{tabular}{|l|c|c|c|c|}
\hline & \multicolumn{2}{|c|}{ URUGUAYOS } & \multicolumn{2}{c|}{ EXTRANJERo } \\
\hline & HoMBres & MUjerES & HOMBRES & MUJERES \\
\hline $\mathbf{1 8 5 8}$ & 29 & 11 & 78 & 19 \\
\hline $\mathbf{1 9 0 8}$ & 63 & 14 & 97 & 21 \\
\hline
\end{tabular}

Fuentes: Padrón de Montevideo 1858-59 (AGN) y Censo Nacional de 1908.

Durante la segunda mitad del siglo XIX, la población aumentó debido a una reducción en el número de guerras civiles, mejores condiciones de vida y una gran afluencia de inmigrantes. Los recién llegados se distribuyeron de manera desigual en todo el territorio: su impacto en la población total fue mayor en el sudoeste y alrededor de la ciudad de Montevideo que en el resto del país.

Cuadro 4. Extranjeros en Uruguay, 1858-1908 (en \%)

\begin{tabular}{|l|l|l|l|}
\hline & URUGUAY & INTERIOR & MONTEVIDEO \\
\hline & EXTRANJEROS (\%) & EXTRANJEROS (\%) & EXTRANJEROS (\%) \\
\hline $\mathbf{1 8 5 8 / 5 9}$ & 33,5 & & 44,7 \\
\hline $\mathbf{1 8 8 9}$ & & & 46,8 \\
\hline $\mathbf{1 9 0 8}$ & 17,4 & 11,9 & 30,4 \\
\hline
\end{tabular}

Fuentes: Padrón de Montevideo 1858-59 (AGN), Censo de Población del departamento de Montevideo (1889) y Censo Nacional de 1908.

Gráfico 2 Población de Montevideo por edad y sexo ssegùn nacionalidad. 1858
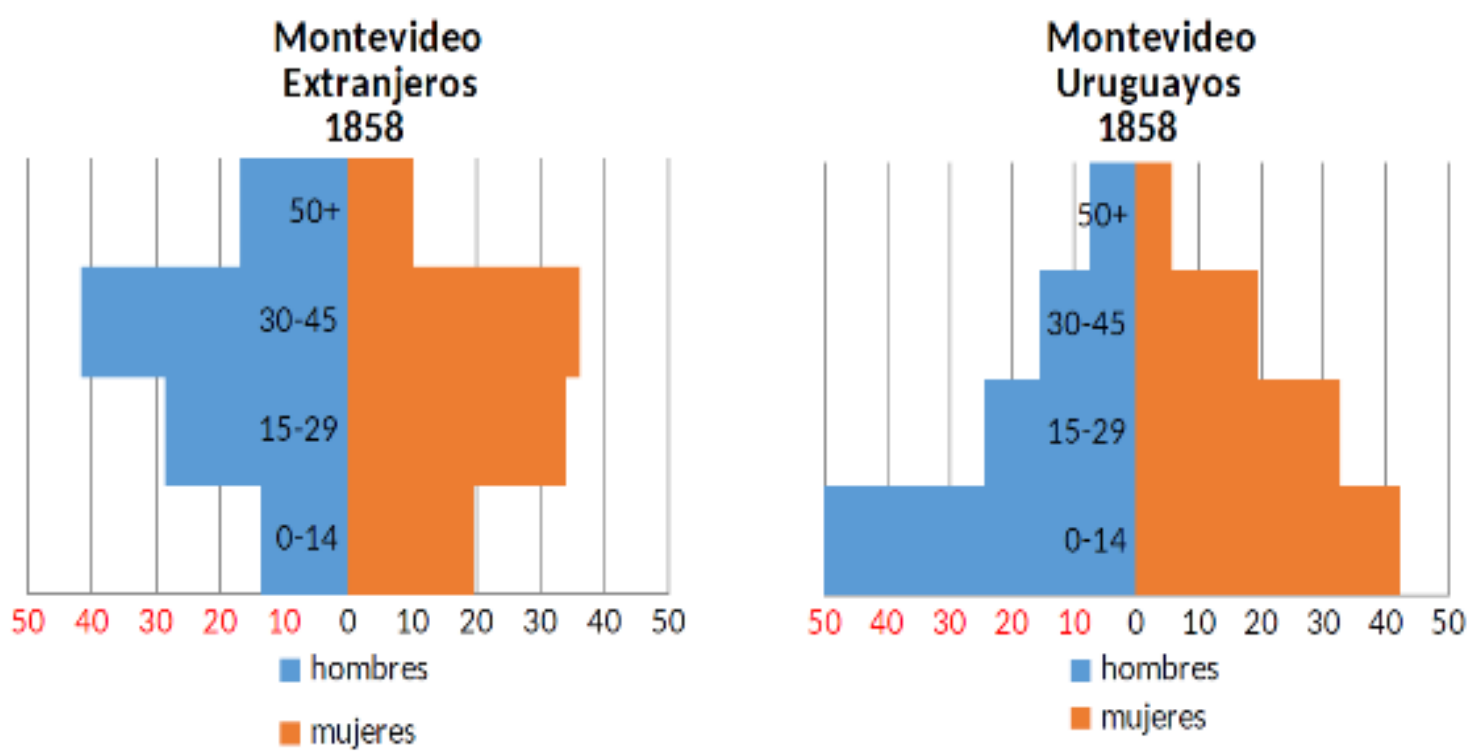

Fuentes: Padrón de Montevideo 1858-59 (AGN)

En la población uruguaya nativa de Montevideo observamos una estructura de edades joven, característica del comportamiento demográfico de una población pretransicional. A esto se suma el impacto de la recientemente terminada guerra civil (1851). La sobre representación femenina de la población uruguaya entre 15 y 50 años, debe estar influida por el impacto de las guerras que afectan más a los hombres. Asimismo, la estructura productiva agraria uruguaya tendió a excluir la mano de obra femenina y aumentar la migración femenina de las zonas rurales a las urbanas. 
Gráfico 3 Población de Montevideo por sexo y edad en 1908.

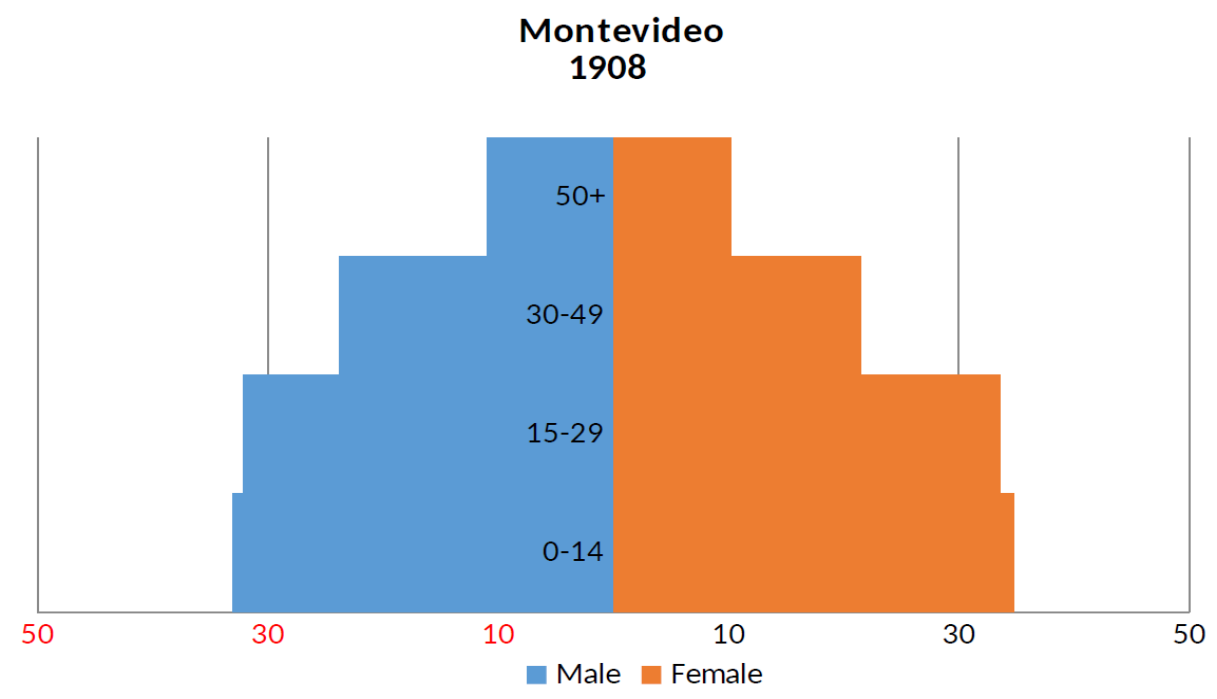

Fuentes: Censo Nacional de 1908

Cincuenta años después, la pirámide de población había cambiado y mostraba una mayor proporción de niños y una menor concentración en el grupo de edad de la población activa. La sociedad estaba en camino a la transición demográfica.

A principios del siglo XX, Uruguay mostraba junto a Chile y Argentina, una tasa relativamente alta de participación de las mujeres en el mercado laboral, muy superior a la de Brasil, Colombia y México (Camou 2016). La tasa de participación femenina de las extranjeras en la fuerza laboral fue mayor a la de las uruguayas a lo largo del período. De 1889 a 1908, la participación femenina en el mercado de trabajo disminuyó para ambos grupos. Esta tendencia puede vincularse a un patrón más global de la evolución del trabajo remunerado femenino, constatado para otros países, que registra mayores niveles de participación femenina en las primeras etapas de desarrollo, seguido de una disminución durante el período de rápida industrialización (Goldin 2006, Seguino and Grown 2006, Camou 2016).

\section{Las relaciones de trabajo en Uruguay}

La consolidación de un mercado laboral asalariado aparece como un proceso gradual que tiene lugar en el período en estudio. A fines del siglo XIX y principios del XX, la economía uruguaya estaba experimentando grandes cambios derivados de su integración en la economía internacional. El sector exportador, apoyado por la modernización del transporte, impulsó el auge económico. El desarrollo de una economía urbana generó una mayor demanda de mano de obra en la manufactura y los servicios.

Las estimaciones realizadas de acuerdo a la taxonomía de Global Project dan cuenta de este proceso. Desde 1858 hasta 1908, se produce un aumento importante de los asalariados en estas décadas. Como parte de esta transformación de la economía, el peso relativo de los trabajadores autónomos y los empleadores disminuyó. La etapa se corresponde con el establecimiento de las primeras grandes fábricas textiles, cervecerías, saladeros y frigoríficos, etc. Como consecuencia de este proceso de asalarización disminuyó la proporción de personas sin ocupación declarada (familiares productores y no productores). 
Gráfico 4. relaciones de trabajo, Montevideo 1858 y 1908

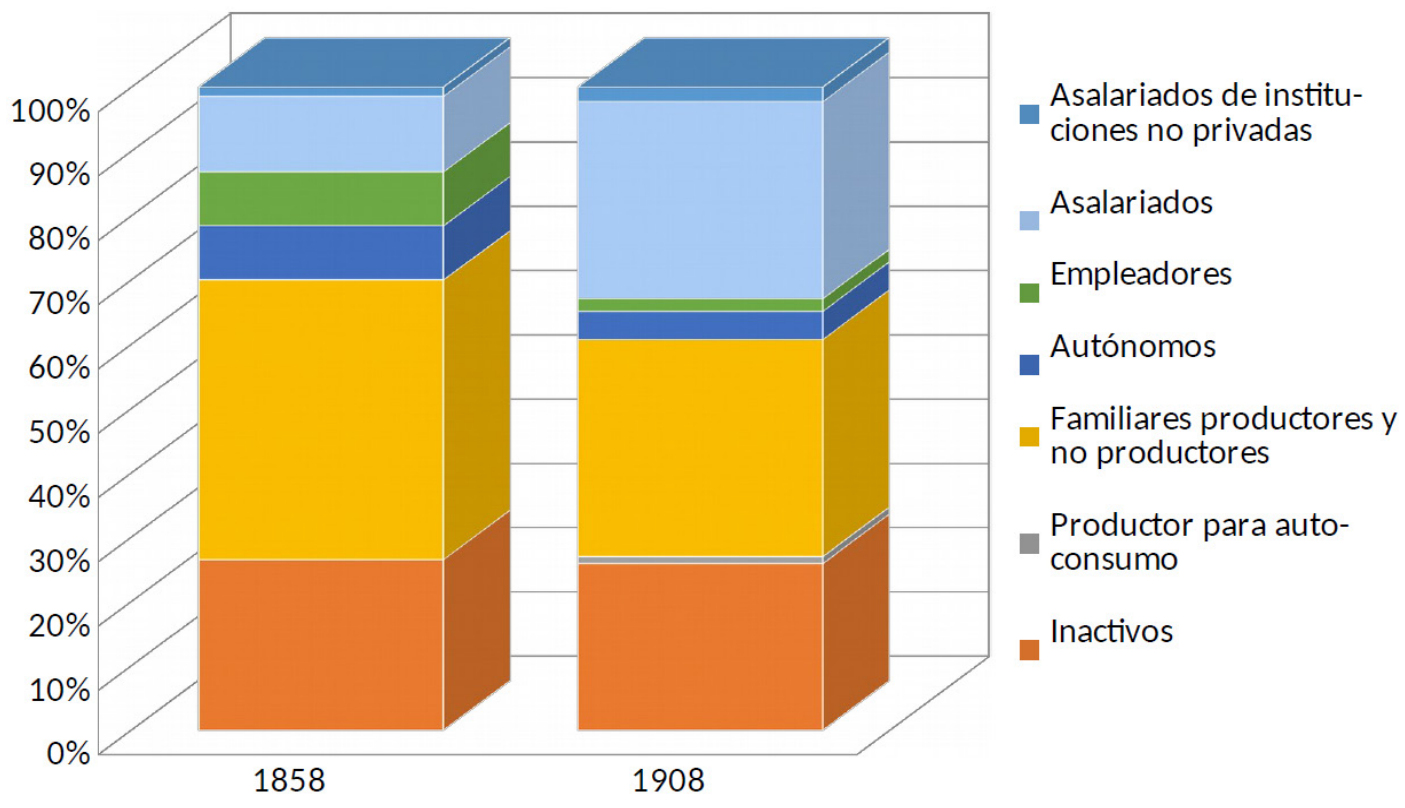

Fuentes: Padrón de Montevideo 1858-59 (AGN) y Censo Nacional de 1908.

Montevideo, como el principal polo de atracción para los inmigrantes en el país, experimentó importantes cambios en la composición y el volumen de la población. Uno de los cambios más importantes fue la integración masiva de inmigrantes a las actividades productivas.

Entre 1858/59 y 1908 los inmigrantes disminuyeron como porcentaje de la población activa, aunque continúan representando una proporción importante de la fuerza de trabajo. Esto se explica por un enlentecimiento del ritmo de llegada de inmigrantes. Por otra parte de acuerdo a la legislación uruguaya, sólo la primera generación puede tenerse en cuenta. Asimismo, los nacionales tendieron a aumentar su participación en la población activa a lo largo del periodo, como consecuencia de la finalización de las guerras civiles y el aumento de la demanda de trabajo. Aun así, para ambos puntos de referencia, los inmigrantes aparecen sobre representados en casi todas las categorías laborales, especialmente entre los asalariados privados.

Cuadro 5. Extranjeros por categoría ocupacional (\%). Montevideo

\begin{tabular}{|l|c|c|c|c|}
\hline & \multicolumn{2}{|c|}{1858} & \multicolumn{2}{c|}{1908} \\
\hline & $\%$ & \multicolumn{1}{|c|}{ DIF* $^{*}$} & $\%$ & DIF $^{*}$ \\
\hline INACTIVOS & 21 & -26 & 6 & -24 \\
\hline PRODUCTOR PARA AUTOCONSUMO & & -48 & 55 & 24 \\
\hline FAMILIARES PRODUCTORES Y NO PRODUCTORES & 40 & -8 & 32 & 1 \\
\hline AUTÓNOMOS & 94 & 46 & 39 & 9 \\
\hline EMPLEADORES & 72 & 24 & 55 & 24 \\
\hline ASALARIADOS & 120 & 72 & 46 & 16 \\
\hline ASALARIADOS DE INSTITUCIONES NO PRIVADAS & 38 & -10 & 24 & -6 \\
\hline POBLACIÓN TOTAL & $\mathbf{4 8}$ & & $\mathbf{3 1}$ & \\
\hline
\end{tabular}

Fuentes: Padrón de Montevideo 1858-59 and Censo Nacional de 1908

Dif* Porcentaje de la categoría menos el porcentaje de la población total. 
El proceso de disciplinamiento e incorporación de los hábitos de trabajo "modernos" aún no parecen estar consolidado entre los uruguayos. En 1858, la clase alta declaraba profesiones como "propietario", "comerciante" y "militar", pero la población "sin ocupación" o inactiva ascendía al $82 \%$ de la población (M. Camou and Pellegrino 1992) ${ }^{1}$. Este patrón parece coincidir con el mundo que describe Barrán (2001), en el que el entretenimiento, el juego, la guerra, la política y los grandes negocios eran las actividades principales de los habitantes. Es probable que existiera aún un importante contingente de fuerza laboral inestable e informal.

Gráfico 5. Relaciones de trabajo en Montevideo por nacionalidad.
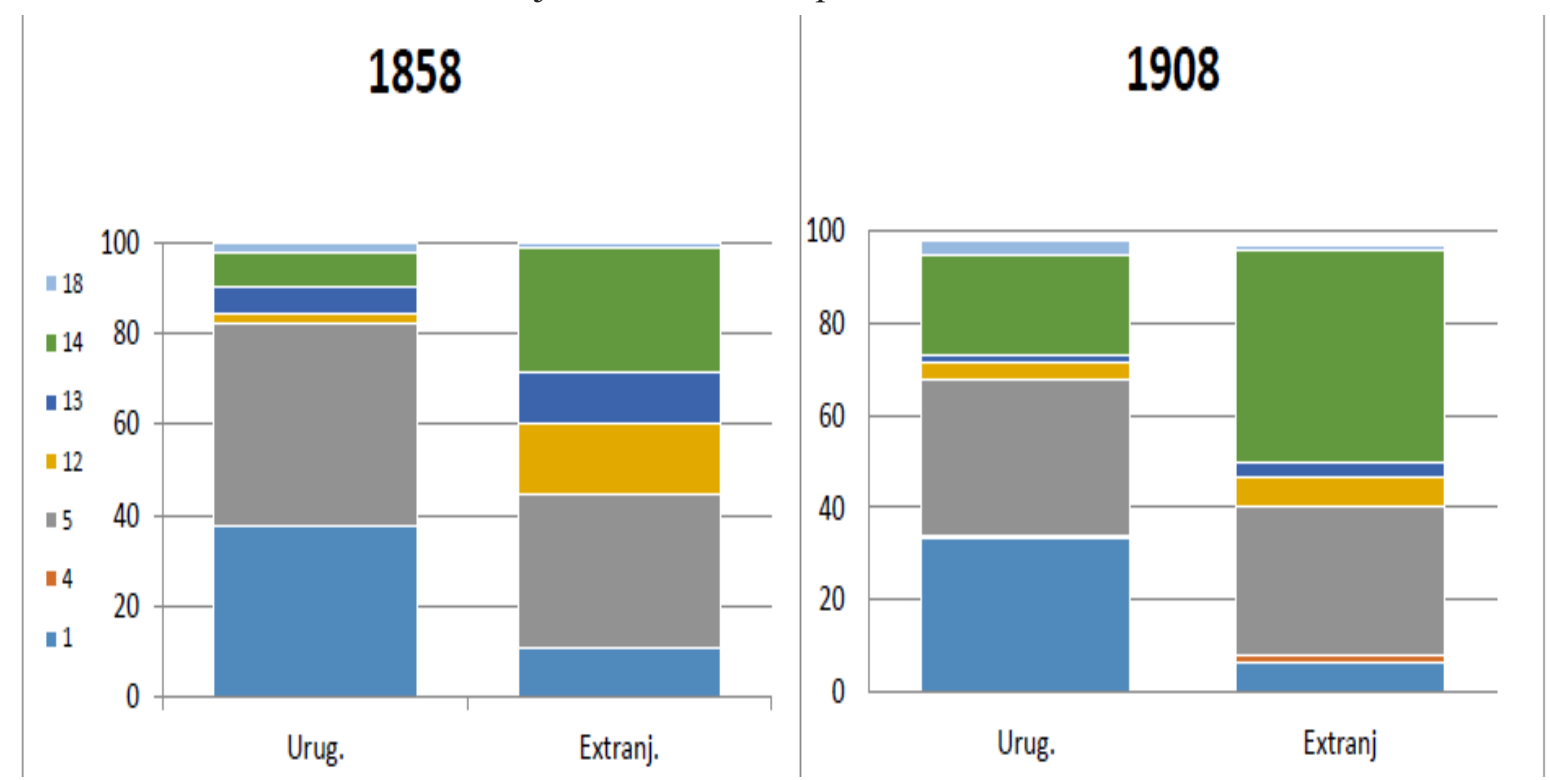

Nota:1: Inactivos, 4: Productor para autoconsumo, 5: Familiares productores y no productores, 12Autónomos, 13: Empleadores, 14: Asalariados, 18: Asalariados de instituciones no privadas.

Fuentes: Padrón de Montevideo 1858-59 and Censo Nacional de 1908

La estructura de las relaciones laborales por nacionalidad también aparece fuertemente determinada por el perfil demográfico de los inmigrantes y los nativos. La baja proporción de extranjeros en la categoría inactiva se explica por su menor fecundidad, el registro de sus hijos nacidos en el país como uruguayos y la concentración de la población en la edad activa.

Los extranjeros figuran sub representados solamente en el grupo de asalariados en instituciones fuera del mercado, que comprende principalmente a los empleados públicos. Aunque los inmigrantes recién llegados fueron contratados como jornaleros para la construcción de los servicios públicos, no estaban incluidos en la categoría de funcionarios públicos.

Los extranjeros se concentraron más fuertemente también en las categorías de empleadores y autónomos, afirmando el supuesto de que se trata de un grupo de población con mayor iniciativa empresarial y movilidad social.

En 1908, la participación de los uruguayos en la categoría de asalariados aumentó, pero aún continuó siendo muy inferior al patrón prevaleciente entre los extranjeros.

Desde las últimas décadas del siglo XIX, la cobertura de la educación primaria se extendió en Uruguay y especialmente en Montevideo, seguida por una rápida disminución de la tasa de analfabetismo. Nos preguntamos si el nivel educativo puede ser un factor explicativo de las diferencias en la inserción en el mercado de trabajo entre inmigrantes y nacionales.

1 En esta investigación se realiza una estratificación social de la población tomando en cuenta la profesión, la ubicación de la vivienda, la conformación familiar y la presencia de servicio domésticoy ex esclavos en el hogar. 
Cuadro 6. Analfabetismo por nacionalidad $(15+)$ Montevideo.

\begin{tabular}{|c|c|c|c|c|}
\hline & \multicolumn{2}{|c|}{ URUGUAYOS } & \multicolumn{2}{|c|}{ EXTRANJEROS } \\
\hline & HOMBRES & MuJERES & HOMBRES & Mujeres \\
\hline 1899 & 34,1 & 32,6 & 34,7 & 51,0 \\
\hline 1908 & 16,8 & 16,3 & 22,4 & 38,9 \\
\hline
\end{tabular}

Fuentes: Censo de Población del departamento de Montevideo de 1889 y Censo Nacional de 1908.

Algunos investigadores han sostenido que la inmigración tuvo un impacto postivo en el nivel educativo en América Latina (Manzel, Baten et al. 2012). Para Uruguay no se constata este efecto, los niveles educativos de los inmigrantes son similares a los uruguayos para los hombres e inferiores para las mujeres.

Por otra parte, estos datos reflejan que los inmigrantes llegados al país tenían un nivel superior al promedio de sus regiones de origen. En Uruguay entre 1860 y 1880 el $65 \%$ de los inmigrantes italianos provenían de las regiones de Liguria, Campania y Basilicata ${ }^{2}$ donde los niveles de analfabetismo eran en torno al $80 \%$ para los hombres y el $90 \%$ para las mujeres (Langeli and Toscani 1991).

De los inmigrantes españoles el 90 \% provenía de Galicia, Cataluña y el País Vasco entre 1860 y 1880 (M. M. Camou 1997), un censo de 1877 presenta niveles de analfabetismo en torno al $50 \%$ de los hombres y entre 60 y 90\% para las mujeres para esas regiones (Espigado Tocino 1990).

En el contexto de la rápida disminución del analfabetismo, la diferencia puede explicarse en parte por el aumento en el número de adultos en sus edades medias y mayores de cincuenta entre los extranjeros. La brecha de género de los extranjeros es más alta que la de los uruguayos y sigue el mismo patrón de sus países de origen (Sarasúa García 2002).

Cuadro 7. Distribución de la población por grupos de edad (15+). Montevideo (\%)

\begin{tabular}{|l|c|c|c|c|}
\hline & UruguaYos & & EXTRANJEROS & \\
\hline & HoMBres & MUjERES & HoMBres & MujERES \\
\hline $\mathbf{1 5 - 2 9}$ & 28,7 & 31,3 & 20,3 & 20,3 \\
\hline $\mathbf{3 0 - 4 9}$ & 15,8 & 16,3 & 36,7 & 34,6 \\
\hline $\mathbf{5 0 +}$ & 5,1 & 5,5 & 35,5 & 33,4 \\
\hline
\end{tabular}

Fuentes: Censo Nacional de 1908.

De todas maneras, se puede argumentar que existió un vínculo positivo indirecto entre educación y migración o a través de externalidades vinculadas a los conocimientos aportados desde sus sociedades de origen como lo señalan Bértola y Ocampo (2012). A su vez, los niveles educativos comparativamente altos de Uruguay respecto a la región constituyeron un factor de atracción. Existe también una agenda de investigación que vincula la migración y la diversidad cultural como un factor que favorece el desarrollo y el crecimiento.

\section{A modo de conclusión}

El artículo presenta una caracterización de las relaciones de trabajo para países del Cono Sur y Bolivia a comienzos del siglo XX.

En un primer paso, nuestro objetivo es comparar los principales cambios entre países en la etapa de conformación del mercado de trabajo moderno.

Los resultados reflejan importantes diferencias entre las estructuras de la ocupación de estos países. La evidencia recolectada permitió comparar cuantitativamente indicadores del tipo de relaciones de

2 Datos de expedientes matrimoniales relevados por la autora y A. Pellegrino. 
trabajo de los distintos países en el período de inserción en la economía internacional. Encontramos que Bolivia es un país que se encuentra en una etapa pre moderna, Brasil presenta una estrucutra intermedia y Argentina y Uruguay han evolucionado hacia un mercado de trabajo con importantes sectores asalariados.

La no inclusión de información sobre las dimensiones raciales de la población en los censos latinoamericanos del período no permite establecer conclusiones firmes, pero las diferencias en la composición étnica de las poblaciones en cuestión parece haber jugado un factor relevante. Argentina y Uruguay fueron los países más étnicamente homogéneos con un gran número de inmigrantes europeos, mientras que Brasil y Bolivia fueron más heterogéneos con una alta proporción de poblaciones indígenas o afrodescendientes y una pequeña élite de criollos (personas de ascendencia principalmente europea). Dada la escasa investigación existente sobre este tema desde una perspectiva histórica, creemos que esta caracterización de las relaciones de trabajo aporta una primera mirada sobre estas diferencias.

Analizar el caso de Uruguay por separado, nos permite ahondar en la correspondencia entre los dos conjuntos de variables: las de las relaciones laborales y las demográficas y llegar a algunas conclusiones sobre sus conexiones, aunque no podamos decir nada definitivo sobre su causalidad.

La sociedad montevideana experimenta fuertes transformaciones de las relaciones de trabajo. Nuestros resultados muestran una estructura poblacional muy atípica debido al impacto de la inmigración. Asimismo, revela a nuestro entender distintos niveles, ritmos y sectores de participación en el mercado de trabajo entre uruguayos y extranjeros. La variable educación formal no conforma un argumento importante para explicar estas diferencias, pero podemos suponer que tanto las características demográficas como otro tipo de saberes y trayectorias personales influyen en la forma en que estos dos grupos se integran al mercado de trabajo. Esperamos que, con estos primeros resultados como punto de partida, esta investigación pueda avanzar hacia un estudio de casos particulares y un examen de cómo los cambios demográficos afectan las relaciones laborales y viceversa.

\section{Bibliografía y fuentes}

\section{Fuentes}

Países latinoamericanos de la muestra: https:/iisg.amsterdam/en/research/projects/globalcollaboratory-on-the-history-of-labour-relations-1500-200

Uruguay. 1908. Censo General de La República En 1908. Vol. T.II, Part. Montevideo: Anuario Estadístico de la República Oriental del Uruguay.

Uruguay. Censo de Población del departamento de Montevideo de 1889

Urruguay. Padrón de Montevideo 1858-59. Archivo General de la Nación

\section{Referencias bibliográficas}

Barrán, J. P. (2001). Historia de la sensibilidad en el Uruguay: La cultura" bárbara"(1800-1860), Ediciones de la Banda Oriental.

Bértola, Luis (2000). Income Distribution and the Kuznets Curve: Argentina and Uruguay since the 1870s. Second LACLIO Conference, Stanford University.

Bértola, L., et al. (2010). Human Development and Inequality in the 20th Century: the Mercosur Countries in a comparative perspective. Living Standards in Latin American History. Height, Welfare and Development, 1750-2000. R. Salvatore, J. Coatsworth and A. Challú. Harvard, Harvard University Press.

Bértola, L. and P. Gerchunoff (2011). "Institucionalidad y desarrollo económico en América Latina." Santiago de Chile, Chile: Naciones Unidas.

Bértola, L. and J.A. Ocampo (2012). The economic development of Latin America since independence. Oxford, Oxford University Press.

Bulmer Thomas, V. (2003). The Economic History of Latin America since Independence. New York, Cambridge University Press.

Camou, M. and A. Pellegrino (1992). Una fotografía instantánea de Montevideo. Ediciones del 
Quinto Centenario. U. d. 1. República. Montevideo

Camou, M M. 1997. "Volumen y características demográficas de la inmigración española." En: Españoles en el Uruguay, Carlos Zubillaga (Ed.), 53-92. Montevideo: Universidad de la República. Facultad de Humanidades y Ciencias de la Educación

Camou, M.M (2016) "Historical Patterns of Gender Inequality in Latin America: New Evidence" en: Camou, M.M, Maubrigades, S.\& Thorp, R. (Eds.): Gender Inequalities and development in Latin America during the Twentieth. Londres, Ashgate-routledge.

Gil Montero, R. n.d. "Methodological Paper Bolivia 1900-2000.” Amsterdam. https://collab.iisg.nl/ web/LabourRelations/.

Grantham, G. \& MacKinnon, M. (1994). Introduction to Labour market evolution: The economic history of market integration, wage flexibility, and the employment relation. London: Routledge.

Goldin, C. (2006). "The Quiet Revolution That Transformed Women's Employment, Education, and Family." The American Economic Review 96(2): 1-21.

Hatton, T. J. and J. G. Williamson (1994). International Migration 1850-1939: An Economic Survey. Migration and the International Labor Market, 1850-1939. T. J. Hatton and J. G. Williamson, Routledge: pp. 3-35.

Langeli, B. y Toscani, X. (1991). "Prologo: Istruzione, alfabetismo, scrittura.sagi di storia dell'alfabetizzacione in Italia (sec.XV-XIX)". Milán. Franco Angeli.

Lucassen, J.O. (2013). Outlines of a History of Labour. IISH-Research Paper 51.

Manzel, K; Baten,J. z Stolz, Y. (2012). "Convergence and divergence of numeracy: the development of age heaping in Latin America from the seventeenth to the twentieth century". Economic History Review, 65, 3, pp. 932-960

Otero, H. 2011. "El Concepto de Población En El Sistema Estadístico de Argentina, 1869-2001." Estatística e Sociedade No.1: 7-25.

Pellegrino, A. (2013). "Uruguay: cien años de transición demográfica." Migración y Desarrollo 11, Nr.20.

Pérez, Alexis Torrico. (2017). "Bolivia En El Siglo XIX y Su Desempeño Económico (1880-1910)." In Un Siglo de Economía En Bolivia 1900-2016 Tomo II, en: Castellanos Velásquez, I. pp. 429-62. La Paz: Konrad Adenauer Stiftung.

Sábato, H. and L. A. Romero (1992). Los trabajadores de Buenos Aires: la experiencia del mercado: 1850-1880. Buenos Aires.

Sanchez Alonso. 2006. "Labor and Inmigration" In The Cambridge Economic History of Latin America., edited by V. Bulmer-Thomas, Coatsworth J., and Cortés Conde R. New York: Cambridge University Press.

Sarasúa, C. (2002) "El acceso de niñas y niños a los recursos educativos en la España rural del siglo XIX” en: Carrión, J.M.(Ed.). El Nivel de Vida en la España Rural, Siglos XVIII-XX. Universidad de Alicante.

Stephanie Seguino \& Caren Grown. (2006). "Gender equity and globalization: macroeconomic policy for developing countries," Journal of International Development, John Wiley \& Sons, Ltd., vol. 18(8), pages 1081-1104. 